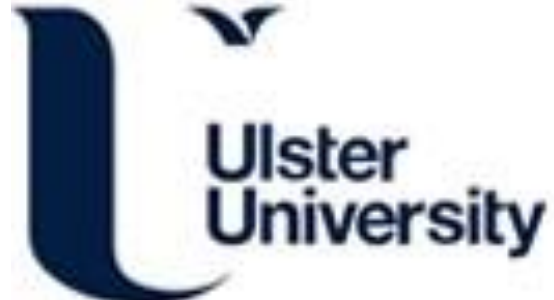

\section{Approaching long term cardiac rhythm monitoring using advanced arm worn sensors and ECG recovery techniques.}

Lynn, WD., Escalona, OJ., \& McEneaney, D. (2015). Approaching long term cardiac rhythm monitoring using advanced arm worn sensors and ECG recovery techniques. Cardiology and Angiology: An International Journal, 3(3), 137-148. https://doi.org/10.9734/CA/2015/16768

Link to publication record in Ulster University Research Portal

Published in:

Cardiology and Angiology: An International Journal

Publication Status:

Published (in print/issue): 15/04/2015

DOI:

10.9734/CA/2015/16768

Document Version

Publisher's PDF, also known as Version of record

\section{General rights}

Copyright for the publications made accessible via Ulster University's Research Portal is retained by the author(s) and / or other copyright owners and it is a condition of accessing these publications that users recognise and abide by the legal requirements associated with these rights.

\section{Take down policy}

The Research Portal is Ulster University's institutional repository that provides access to Ulster's research outputs. Every effort has been made to ensure that content in the Research Portal does not infringe any person's rights, or applicable UK laws. If you discover content in the Research Portal that you believe breaches copyright or violates any law, please contact pure-support@ulster.ac.uk. 


\title{
Approaching Long Term Cardiac Rhythm Monitoring Using Advanced Arm Worn Sensors and ECG Recovery Techniques
}

\author{
William D. Lynn ${ }^{1 *}$, Omar J. Escalona ${ }^{1}$ and David J. McEneaney ${ }^{2}$ \\ ${ }^{1}$ University of Ulster, School of Engineering, Engineering Research Institute, Newtownabbey, \\ BT37 OQB, UK \\ ${ }^{2}$ Craigavon Area Hospital, Southern Health and Social Care Trust, Cardiac Unit, SHSCT, \\ Portadown, BT63 5QQ, UK.
}

\begin{abstract}
Authors' contributions
This work was carried out in collaboration between all authors. Author WDL designed the study, managed the literature searches and wrote the first draft of the manuscript. Author OJE managed the experimental process. Author DJM wrote the protocols and the ethical approval application. All authors read and approved the final manuscript.

Article Information

DOI: $10.9734 / C A / 2015 / 16768$ Editor(s):

(1) Francesco Pelliccia, Department of Heart and Great Vessels, University La Sapienza, Rome, Italy. Reviewers:

(1) Anonymous, Qatar.

(2) Anonymous, Italy.

(3) Anonymous, China.

(4) Anonymous, India. Complete Peer review History: http://www.sciencedomain.org/review-history.php?iid=842\&id=26\&aid=8835
\end{abstract}

Review Article

Received $12^{\text {th }}$ February 2015 Accepted $28^{\text {th }}$ March 2015 Published $15^{\text {th }}$ April 2015

\begin{abstract}
According to recent British Heart Foundation statistics, one in six men and more than one in ten women die from coronary heart disease (CHD) in the UK. This equates to almost 74,000 deaths per annum from CHD alone. More worryingly, every week, 12 apparently fit and healthy young people aged 35 and under, die from undiagnosed cardiac conditions. In both circumstances, monitoring is preformed only when triggered by an event. Unfortunately, this may be too late in the large majority of cases. For instance, there is evidence suggesting that most indiscernible cardiac abnormalities are made detectable by ECG through the act of suddenly standing upright. This infers that the condition would be detectable during the course of everyday ambulatory activity and highlights the need for a long term monitoring device. Current diagnostic equipment consists of the Holter monitor
\end{abstract}


for extended periods up to 36 hours and the implantable loop recorder (ILR) for monitoring up to 3 years. The diagnostic yield of the ECG monitoring strategy is greatly increased as the monitoring period increases. Therefore, for subjects that exhibit symptoms of cardiac involvement that are transient in nature, the ILR offers the best opportunity for diagnosis. However, the ILR is inserted under the surface of the skin in the upper chest area and requires a surgical procedure, with associated risks, which makes ILR's a costly and inconvenient option in many cases.

The need for a non-invasive long term monitoring device, which is comfortable to wear along the arm and able to provide reliable ECG monitoring, has been addressed by many, in several lines of approach to a solution. This review details the current state of the art and any pending limitations. It then presents key multidisciplinary solutions on the different aspects of the problem, which will still require integration in order to realise such a device.

Keywords: ECG; long term; monitor; arm worn; dysrhythmia.

\section{INTRODUCTION}

As heart disease remains a prevalent killer of both the old and the young [1], there is a strong case for the development of a long term monitoring technology that is non-invasive and comfortable to wear. Also, with recent research alluding toward an increased detectability of latent cardiac abnormalities once the subject stands from a seated position [2], the desirability and marketability of an arm worn sensor is increasingly realised [3].

The effect of drugs on the heart rhythm is widely noted in the literature. An example being those which are linked with QT elongation or torsade de pointes. Domperidone, for instance has been associated with a small increased risk of serious ventricular dysrhythmia or sudden cardiac death $[4,5]$. A warning strategy for patients continuously monitored during their course of treatment would significantly de-risk the administration period.

Atrial fibrillation (AF) affects almost 800,000 people in the UK, predominantly over 75 year olds with an affect rate of $10 \%$. AF is uncommon in younger people; however, it may be coincidental with other heart defects such as heart valve problems [6]. Gauging the time a patient spends in paroxysmal atrial fibrillation will determine the treatment plan. Current analysis and recording equipment, such as the Omron HCG801 [7] (Fig. 1 (a)), do not give a good temporal picture because the recording is not continuous. The Holter monitor (Fig. 1 (b)) is a continuous data storage device; however, it is a short term technology, offering recording over time periods of $24 \mathrm{hrs}$ to 2 weeks. For longer recording periods, in the region of 28 days, an External Loop Recorder or Event Loop Recorder (ELR) may be selected by the clinician to act as a diagnostic tool. The ELR (Fig. 1 (c)) is available in two forms - permanently worn (looping memory) or as a post applied recorder (nonlooping memory). The permanently worn device may be automatically triggered by a threshold crossing of the patient's heart rate or patient triggered once he has become aware of a dysrhythmia. The post event recorder is not worn continuously and as a result does not store any historical precursor to the dysrhythmic event. The post event recorder depends on the patient's compliance with general operational protocols, the patient remaining sufficiently coherent to operate the event trigger and the maintenance of consciousness during the scope of the dysrhythmia.

Real time monitoring of the cardiac output can be achieved using a fusion of ELR and cellular phone technology in the form of the Mobile Cardiac Output Telemetry recorder (MCOT) [8] (Fig. 1 (d)). MCOT recorders are a recent development and provide the wearer with a $24 \mathrm{hr}$ full, high resolution data recording device. The device can provide the clinician with real time, streamed data over a cellular interface, hence delimiting the storage capacity. The technology may also be configured as a looping, nonlooping, event triggered and patient triggered device, capable of delivering a recording directly to the clinician.

The Implantable Loop Recorder (ILR) overcomes patient noncompliance through surgical intervention. A small device, similar in size to a USB memory stick (Fig. 1 (e)), is implanted under the skin on the wall of the upper chest. The ILR is event triggered or can be patient triggered by placing an activator proximally on the chest. The more recent generations of this device are designed to operate in excess of 2 years [9] and have wireless communication capability offering the provision of fast delivery of 
data for interpretation and subsequent clinical decision.

Thus, depending on the device time application, the available technology can operate in three time-range categories: limited to short term monitoring, up to 1 month, or very long term, in excess of 2 years. Without the risk and cost of the surgically implanted ILR, the middle category is not catered for. It is also notable that a continuous full data recording device is not yet available for periods over 1 month.

Several key factors influence the success of a proposed very long term monitor. Firstly, the comfort of such a monitor is paramount. The ambulatory patient must be virtually unaware of the monitor's presence to ensure that the urge to tamper with, or completely remove the device prematurely is mitigated. Several authors have suggested comfort sites for wearable electronics $[10,11]$. Gemperle et al. and Shackel et al. discuss the many factors that contribute to a subject feeling comfortable. Shackel discusses ergonomics of work place design, specifically chair comfort while Gemperle tested the design of wearable forms by studying subjects while they perform activities. Each subject rated the forms perceived comfort to allow the construction of a body position comfort model - shown in Fig. 2.

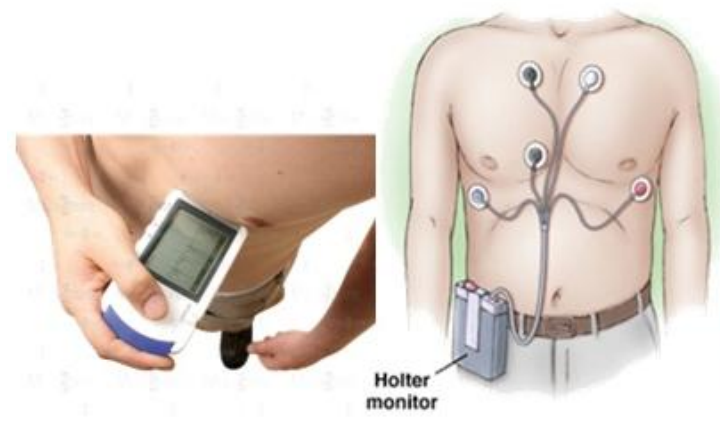

(a)

(b)

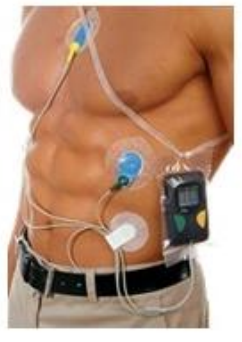

(c)
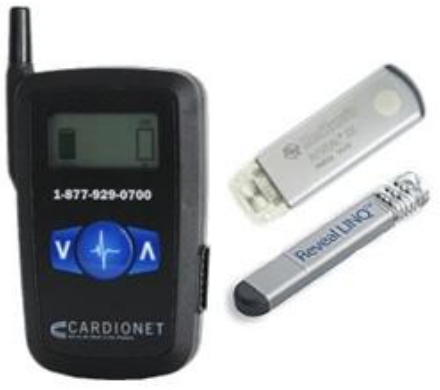

(d)

(e)

Fig. 1. (a) Omoron HCG801 post applied non-looping recorder; (b) standard short term holter recording device; (c) Event Loop Recorder (ELR); (d) Mobile Cardiac Output Telemetry(MCOT); (e) Implantable Loop Recorder (ILR), standard and wirelessly enabled
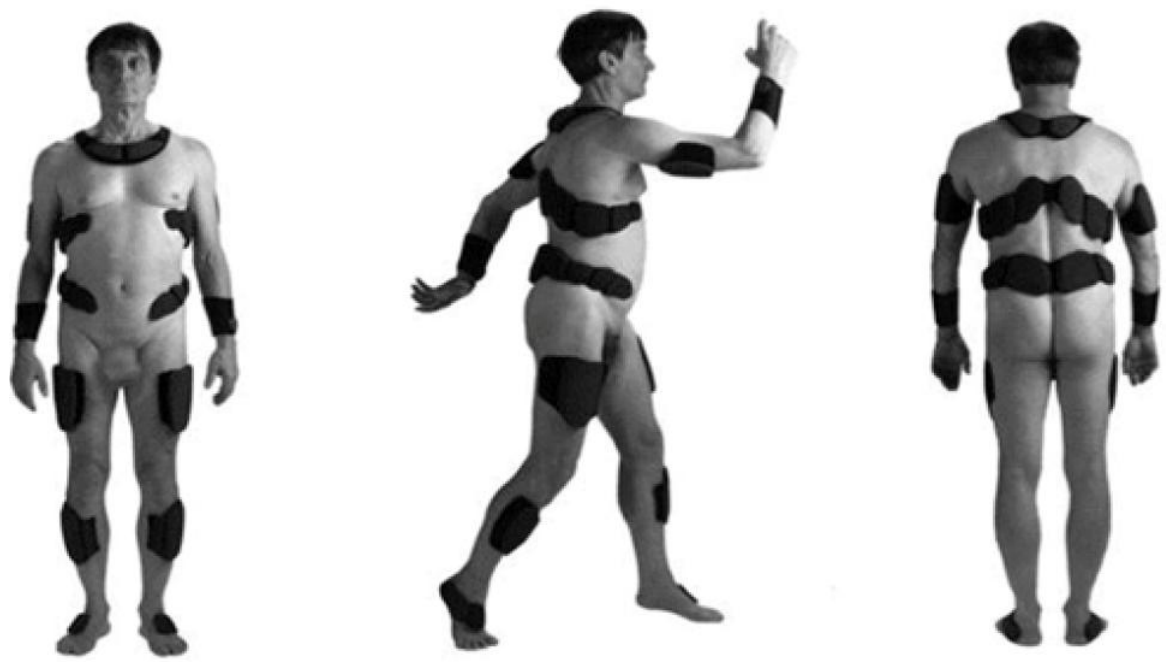

Fig. 2. Body sites for comfortable "wearability" (Gemperle et al.) [4] 
Lynn (2013). concluded, by analysis of the data from a clinical study, that a significant reduction in ECG signal occurred on the right arm [12], an opinion supported by Hung-Chi Yang et al [13]. Hence all attempts at a successful arm worn device refer to data collected on the left limb. Yang developed a flexible foil electrode that can be conveniently wrapped around a limb and will facilitate the recording of ECG information Fig. 3. He successfully extracted QRS information from the upper left arm (bicep region) and elbow position. However, attempts to recover ECG data from the wrist were unsuccessful. Yang has declared that the ECG signal at the wrist is "very weak" and is easily disrupted by electromyographic (EMG) noise. It is also notable that the signal processing used during his study was simplistic, consisting of bandwidth narrowing to remove power line and EMG noise. Yang's chosen noise mitigation technique will have suppressed desired signal information ratio metrically with the noise component, as the frequency domain of the desired signal and the unwanted noise component are overlapped. The electrode design is of interest however. It is also probable that the recordings used in the Yang publication are best case selections and that a dry electrode, of the type described in the experiment, will produce large amounts of electrical noise should the subject move.

\subsection{The Noise Issue}

As is the case in most areas of measurement, the decisive boundary of detectability of a weak signal is determined by the presence of unwanted, random signals - or noise. Noise obscures the desired signal and poses a major challenge in the recovery of biological signals.

The problem of noise is not limited to weak, low amplitude signals. The measurement accuracy and repeatability of comparatively large amplitude signals can also be affected by the presence of a noise component. When discussing the recovery of cardiac electrical activity, the signals can be described as low amplitude and low energy [14]. This means that the electrical signal produced by the heart is incapable of driving a low impedence load. The conditions described by physicist Georg Ohm, govern and predict the capability and behaviour of all measurement systems [15]. Consider Fig. 4; the component $Z_{B M}$ simulates the body impedance model, if the measurement system is 'ideal' and has an input impedance of infinity, the maximum amplitude will be measured. If the measurement system impedance is equal to $Z_{B M}$, the measured signal amplitude will appear to be $50 \%$ of that measured from the 'ideal' system. The degradation continues as the input impedance of the measurement system decreases. In order to achieve a reasonable signal amplitude, a measurement system design with an input impedance of $Z_{B M}$ or greater is typically chosen. The trade-off being that the high impedance input also allows any noise component to be readily evident in the measured signal.

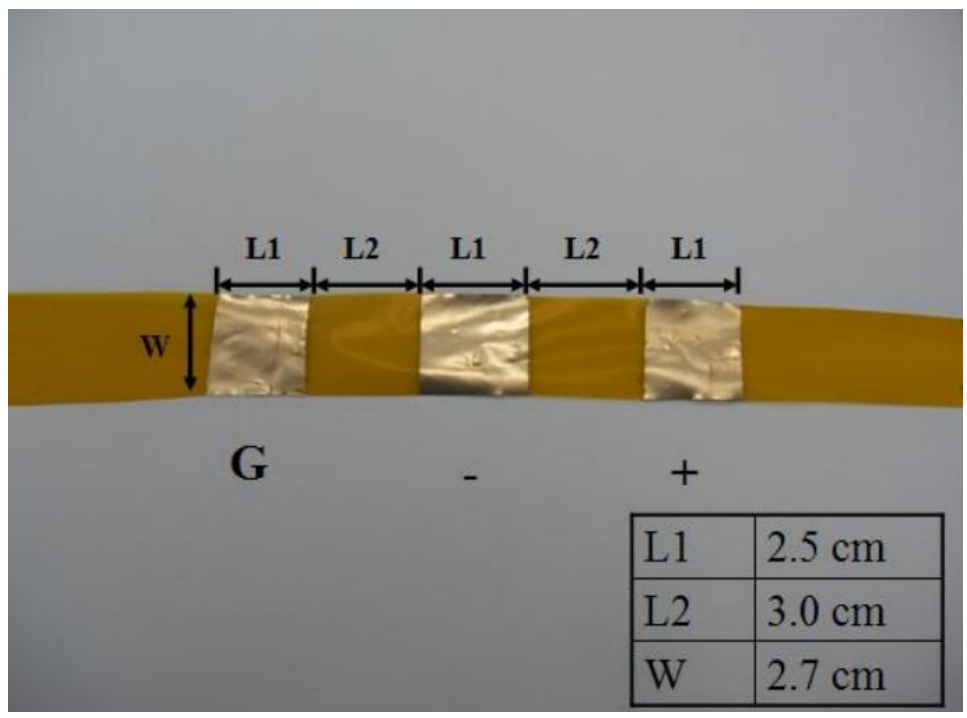

Fig. 3. Flexible printed circuit electrode trialled by Yang $\mathrm{H}$ et al. 2011. [7] 


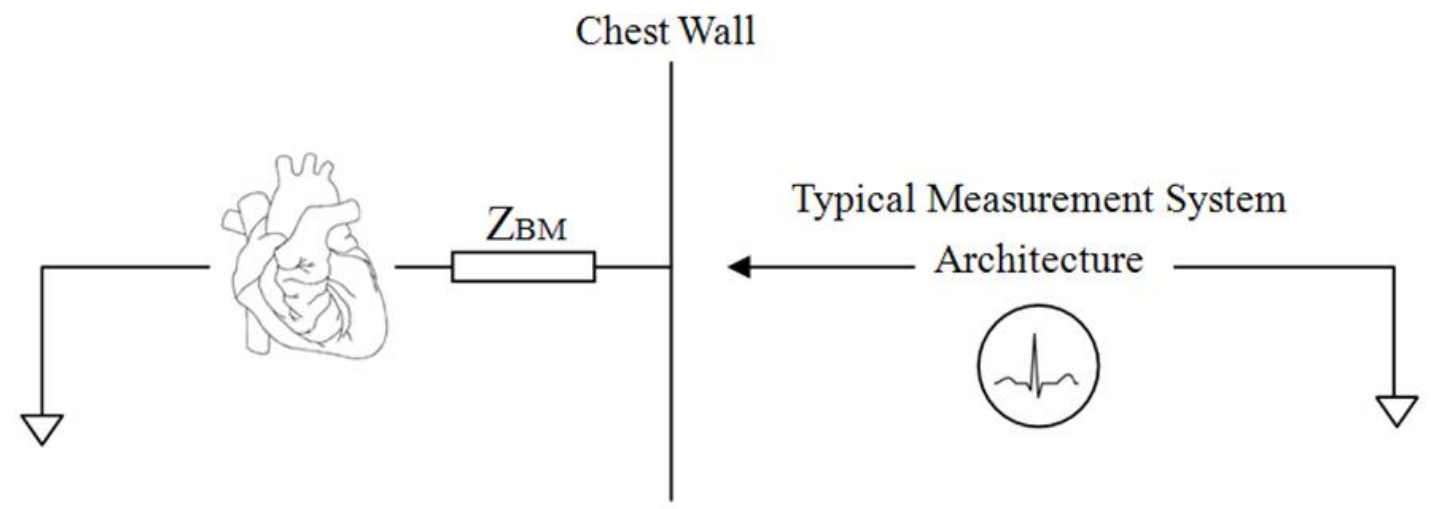

Fig. 4. Illustration showing measurement system interaction with the human body model impedance

Noise falls into two main types - conducted and radiated. Conducted noise is that which is unavoidably present in the final signal. An example of which would be electromyographic disturbances. This type of noise can often be removed by signal averaging or bandwidth narrowing. Radiated noise is that which is propagated through the environment and electromagnetically coupled onto the bioamplifier high impedance input. Radio interference and mains $(50 \mathrm{~Hz} / 60 \mathrm{~Hz})$ interference are examples of this. Radiated noise is often a function of physical layout of the system. Careful positioning of wires, electrodes and power cables can, along with filtering, reduce its affects.

\section{POTENTIAL SOLUTIONS}

\subsection{Signal Denoising Techniques}

\subsubsection{Signal averaging}

Signal averaging (SA) is a reasonably simplistic approach to noise removal from repetitive signals - the ECG, due to its rhythmic nature, is ideal for this approach. The technique requires a fiducial [16] or datum point to be identified. All subsequent repetitions of the signal are temporally aligned and the noise signals are expected to annihilate. The SA technique requires that the noise signals are non-coherent for successful filtering to take place. Power line interference and electromyographic noise sources, through their repetitive nature, often confound this technique.

Temporal misalignment of the fiducial datum has the unwanted effect of integrating the output response, e.g. a pulse stream averaged and processed using an ideal, perfect temporal alignment will reproduce a perfect square pulse as an output. However if the fiducial point is subject to jitter, the output will be a smoothed pulse, ie the high frequency edges will have been lost. This phenomenon was discussed by Escalona et al [16] and William Craelius et al. [17]. Craelius also suggests that a temporal misalignment can add signal components to the filtered signal as well as subtract, producing false triggering of any beat extraction algorithm. A lot can be done to optimise the SA technique and reduce the levels of fiducial point jitter; however, a 'real time' beat to beat comparison is not achievable, therefore a successful clinical implementation of this technique is unlikely.

\subsubsection{Empirical mode decomposition}

Empirical Mode Decomposition (EMD) was developed by N. E. Huang [18] in 1998. EMD uses an iterative process to decompose a signal into a series of Intrinsic Mode Functions (IMF's) subject to the following conditions.

1) In the whole data set the number of extreme and the number of zero crossings must be either equal or differ by, at most, one.

2) At any time the mean value of the envelope of the local maxima and the envelope of the local minima must equal zero.

The IMF's represent the oscillatory modes embedded in the signal $x(t)$. Each IMF is actually a zero mean monocomponent AM-FM signal of the form $x(t)=a(t) \cos \Phi(t)$. As most naturally 
occurring signals contain more than one oscillatory mode they cannot be classed as IMF's. The EMD process is designed to sift through the signal, breaking it down further with each pass, exposing the intrinsic oscillatory modes. The sifting process is defined by Zhao Zhidong et al. [19] as follows:-

Find the local extrema and join the upper $\mathrm{Xup}(\mathrm{t})$ and lower envelopes $\mathrm{Xlow}(\mathrm{t})$ using a smooth cubic spline.

Subtract the mean of the two envelopes $\mathrm{m} 1(\mathrm{t})=$ $((X u p(t)+X \operatorname{low}(t)) / 2)$ to get their difference $h 1(t)$ $=\mathrm{x}(\mathrm{t})-\mathrm{m} 1(\mathrm{t})$.

Repeat steps 1 and 2 until the signal h1(t) meets the criteria set out for an IMF - define as $c 1(t)$. The first IMF contains the highest frequency component of $x(t)$. The residual $r 1(t)$ is given by $r 1(t)=x(t)-c 1(t)$.

Repeat steps 1,2 and 3 using $\mathrm{r} 1(\mathrm{t})$ until all IMF's have been extracted and $r n(t)$ becomes monotonic.

Simply, EMD decomposes a non-stationary signal into narrow band components with decreasing frequency. The original signal is defined as the summation of the IMF's $+\mathrm{rn}(\mathrm{t})$, therefore filtering is achieved by excluding the IMF containing the unwanted information prior to reconstruction by summation.

A comparison of SA and EMD was presented by Lynn et al [20] and showed a promising, low latency method of rhythm artefact extraction from recordings recovered from the upper left arm.

\subsubsection{Ensemble empirical mode decomposition}

A major disadvantage of the classical EMD method is the mode-mixing effect. This becomes evident when pulsed high frequency components are mixed with a lower frequency constant wave. Again the ECG is an example of this type of signal, where the QRS complex is subject to base line drift caused by EMG or $50 \mathrm{~Hz}$ mains. Mode mixing is said to occur when the IMF is not monotonic. This can be overcome by the use of the Ensemble Empirical Mode Decomposition (EEMD).

The EEMD technique [21] overcomes the mode mixing problem by mixing white noise to the residual $(r n(t))$ before each resubmission to the
IMF extraction algorithm. This has the effect of increasing the frequency of the splinic envelope and hence eliminating the aliasing effect which causes mode mixing.

\subsubsection{Wavelet analysis}

An alternative to EMD is the wavelet transform. A method referred to as wavelet transform thresholding is discussed by Alfaouri and Daqroug [22]. The thresholding method is similar to the EMD and EEMD technique, in that it uses the Discrete Wavelet Transform (DWT) to decompose the original into its wavelet series, then it passes the series through a threshold, which removes the coefficients below a certain value, before taking the inverse DWT. The mother wavelet suggested by Alfaouri et al is Daubechies DB4. However, a paper on a similar topic by Singh et al. [23] suggest using DB8. Singh provides evidence based proof for using DB8 and suggests that it produces least root mean square errors (RMSE) and also preserves the peaks of the ECG signal. Alfaouri's work is directed at preserving the lower frequency aspects of the complex, the $\mathrm{P}$ wave and $\mathrm{T}$ wave.

The work carried out by Alfaouri and Singh is directed at denoising and recovery of the ECG complex. Each work has a different agenda but has proved the DWT to be useful in extracting a detailed signal from noise. The technique compares the mother wavelet of choice in both time and scale across the data set and calculates a best fit with the data. This technique can also be used as a syntactic method of QRS detection, as described by $M$. Bahoura et al. [24]. Bahoura's technique is an alternative to nonsyntactic methods such as the Pan-Tomkins [25] method. Non-syntactic techniques tend to suffer from two problems.

1) It has been found that the bandwidth of the QRS may alter between patients and in some cases the same patient at different times.

2) The bandwidth of the noise and the QRS overlap making filtering difficult. As mentioned earlier, this is especially problematic when mains noise at 50 or 60 $\mathrm{Hz}$ is involved. Bahoura's [24] proposed DWT technique is aimed at $P$ and $T$ wave extraction and has been tested on the MIT arrhythmia database. It was suggested that this tool could be used in the post processing of Holter recordings when assessing possible beat arrhythmias. 


\section{LOW NOISE SIGNAL ACQUISITION}

In order to record the ECG signal from remote regions of the body such as the forearm, wrist or thigh, an innovative, low noise signal recovery method will be required to support any signal processing technique. Failure to recover the desired signal is irreversible; no amount of post processing will facilitate extraction.

A wrist worn device that will extract and monitor a clinical quality heart rate and or rhythm is described in US patent 4938228 [26], wrist worn heart rate monitor by William $\mathrm{H}$. Righter et al. Righter, describes several of the essential features required by a wrist worn monitor in his patent.

1) The necessity for the device to be worn on an arm or leg, for example, rather than the chest area.

2) The requirement for the device to utilise a dry electrode arrangement for long term skin contact is highlighted

3) It is suggested that, in order to extract an adequate biometric signal, the acquisition should be in a differential mode.

The Righter patent, although describing an ideal embodiment of the wrist worn clinical ECG monitor, disappoints by underachieving and details claims based around a monitor that requires the application of an oppositely polarised body part, ie the finger of the opposite hand. This creates a significantly large potential across the differential inputs, in the order of 1-2 $\mathrm{mV}$, while the 'true' wrist measured ECG is in the microvolts scale. Righter does not seem to mention the failing of this approach and continues to describe techniques to validate the detection of a QRS complex. His technique is based around maximum coherence matching of the real-time sampled data with a reference waveform. This technique requires that the data to be acted on is sufficiently filtered and free from noise [27].

Any design for a wrist worn monitor that is capable of continuous monitoring of an ECG signal will require analogue signal recovery to be carefully considered. For instance, typical clinical ECG recording equipment is a 'single-ended' recovery system, i.e. each channel is referred to a common point electrode, sometimes referred to as the CMS terminal. This means that the recovered signals are highly susceptible to common mode offset from the electrode potential and to environmental noise. The system also suffers greatly from crosstalk due to the both conducted and radiated noise. In a standard ECG, recording is not noticeably affected. However, when extracting a very low level ECG signal, about one thousand times smaller, from a similar noise environment, then crosstalk can becomes a major issue. A fully differential recovery would provide a solution to handle the effect of crosstalk.

\subsection{Biopotential Amplifiers Solutions}

Many texts describe the use of differential biopotential amplifiers. Nagel, J. H. [28] and Webster, J.G. [29] discuss the basic requirements of a biopotential amplifier and suggest that the minimum criteria are -

The physiological process should not be influenced by the measurement process.

The measured signal should be high fidelity

The amplifier stage should offer high noise rejection

The 'standard' amplifier type used to acquire biological signals is the differential or instrumentation amplifier. This is referenced in the majority of the literature surrounding the topic of instrumentation for biopotential acquisition. There are many variations on the topology from the standard differential conduction amplifiers to non-contact, high impedance amplifiers [30]. Wayne J. Smith et al describe a method of ECG recovery using an innovative, non-contact biopotential electrode. Their technique utilises an OPA129 FET op-amp from Burr-Brown in, what is essentially an open loop gain, differential input. A small portion of negative feedback has been applied to the non-inverting input via a $500 \mathrm{G} \Omega$ resistor to prevent the amplifier from saturating. Smith states that the circuit is a charge amplifier with an overall gain of 2 when the capacitively coupled electrode is about $5 \mathrm{~mm}$ from the body. Smith has quoted noise figures for his design in the order of $70 \mathrm{uV} / \mathrm{VHz}$ to $9.4 \mathrm{uV} / \sqrt{\mathrm{Hz}}$ dependent of frequency. He has suggested an improvement may be made to the system noise figure by changing to an op-amp with a lower bias current and suggests the INA116 device.

Smith's method requires that the measurement system be referenced to the body by means of a contact electrode, therefore is not ideal for a wrist or arm located monitor. However, under some circumstances the technique may be adaptable to be of use. 
Thomas J. Sullivan et al. [31] has developed an integrated sensor specifically for the purpose of EEG and ECG recovery in non-contact mode. Sullivan has used the INA116 device as suggested by Smith [31]. He has highlighted the areas where wet and dry contact electrodes can prove to be unreliable if used for long periods. It is also worth noting that both contact technologies are susceptible to variation in performance due to the wide range of skin types seen in application. This variation is evident across the relatively small sample size recorded in Lynn et al. (2013) clinical study [12], conducted at the Craigavon Area Hospital cardiac research facility.

Sullivan has solved the referencing issue experienced by Smith by using an annular design electrode topology - Fig. 5. The central copper of the electrode layout is a capacitive sense element, as reported by Smith. The guarded output of the INA116 device is used to drive a guard ring that surrounds the central positive electrode. It is also used to drive a shielding layer that is displaced directly behind the capacitive electrode and overlapping the guard ring. The input current is extremely small, $3 f A$, but if left uncontrolled will eventually drive the INA116 into saturation. Smith combats this phenomenon by applying negative feedback. Sullivan has adopted a 'short to ground' approach through an NPN transistor, essentially a periodic reset of the input followed by a period of tolerable drift.
Plessey Semiconductors [32] have developed the Sullivan and Smith approaches and are in the process of launching the EPIC electrostaticvoltometer ASIC in the form of a non-contact ECG electrode. Plessey have also commercialised the EPIC technology as a wrist worn heart monitor [33] but have evidently run into problems of distal location and signal discrimination issues, as the product [34] requires the user to adopt the technique described in the Righter patent [26].

A further alternative to capacitive coupling to allow detection of the changes in the body's electric field, is a proximal impedance spectroscopy technique described in the industrial patent Lynn et al. US0178902A1 [35]. Although the spirit of the patent is biased toward the industrial monitoring of carbon fibre composites, the technique may lend itself to be effective for the detection of small changes in the body's electric field. Essentially, the system uses a plurality of transformer windings, displaced along a continuous ferrite core, to form a magnetic amplifier. The core is swept to determine its resonant frequency. The amplitude and frequency of the natural resonant frequency (F0) are indicative of the instantaneous shunt capacitance (Cp) and shunt resistance (Rp) of the proximal body. It is evident that this technique, although requiring substantial development, may also be capable of detecting an ECG without the requirement for a contact reference electrode.

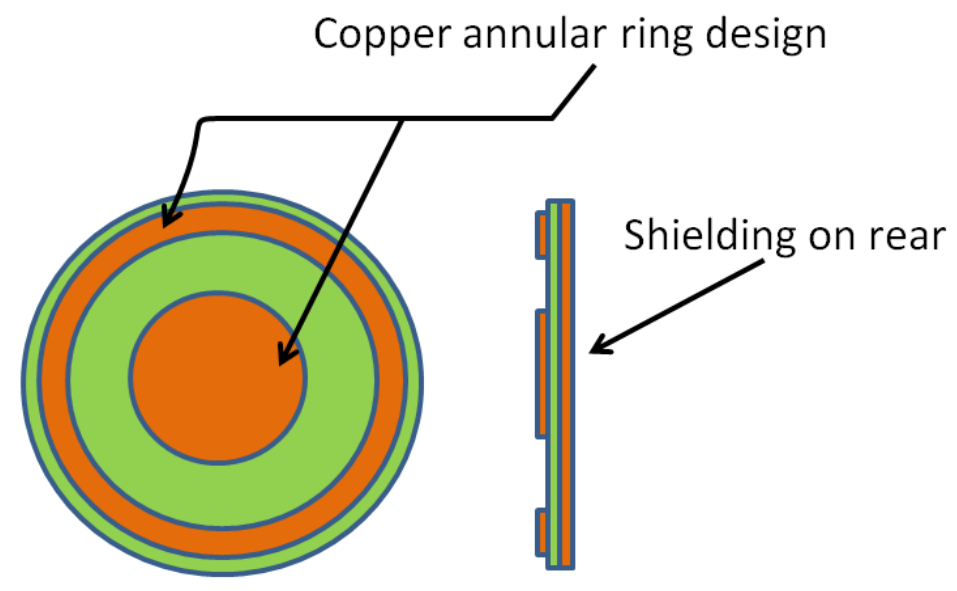

(a)

(b)

Fig. 5. Illustration showing annular ring electrode design cut from FR4 PCB material. (a) frontal elevation. (b) cross-section showing copper shielding layer 


\section{POWERING THE DEVICE}

A body worn device is ultimately required to have a low mass, a problem for long term monitoring. Traditional battery capacity is proportional to mass, as can be seen in Fig. 6 [36]. The portable, body worn device must have a long term operation window in excess of 4 weeks, it must also record and process continuously. All of which requires a significant power delivery capacity. Existing extended period monitoring technologies have circumvented this challenge by either reducing the period where the device is in full operation, by increasing the size of the battery or making the battery replaceable/ rechargeable.

Periodic operation of the system is the usual industry technique for dealing with limited battery capacity. Devices such as implantable loop recorders and pacemakers run, the large majority of their life, in a semi-shutdown mode, only monitoring and processing the heart rhythm. The device is fully powered once an event is detected allowing the device to stay in use for 5 to 10 years [37]. The act of writing to flash memory, delivering a pacing pulse or communicating via a radio frequency link is particularly power hungry and unsustainable for long periods with today's battery technology.
Industry, in particular Supervisory Control and Data Acquisition (SCADA) systems [38], has lead the way on techniques for supporting the limited battery life available for sensing technology. The use of energy harvesting is widespread however, a direct transfer of technology to the biomedical industry is not straight forward. The energy quotients used in industry and those wasted and therefore available for harvesting, are many scales of magnitude greater than those available from the human body. Techniques in kinetic energy harvesting from the body have already been commercialised $[39,40]$; though able to produce considerable energy, are not sufficiently compact or ergonomic to suit long term wearing. Other possibilities are solar [40] and thermoelectric [41], both attractive due to their robustness and high reliability. However, availability of direct light in sufficient quantities for solar operation, traded off against the physical size of the solar panel, is a significant problem. Thermoelectric energy harvested from the human body was first commercialised in the form of the Seiko Thermic wrist watch in 1998 [42]. It is possible to recover approximately $1 \mathrm{~mW}$ of the 100W available from an adult at rest using today's technology. Generation is dependent on a thermal gradient between the skin contact and the outer surface of the thermoelectric generator, an issue if the device is worn under clothing.

\section{Capacity v's Mass of the Panasonic CR series}

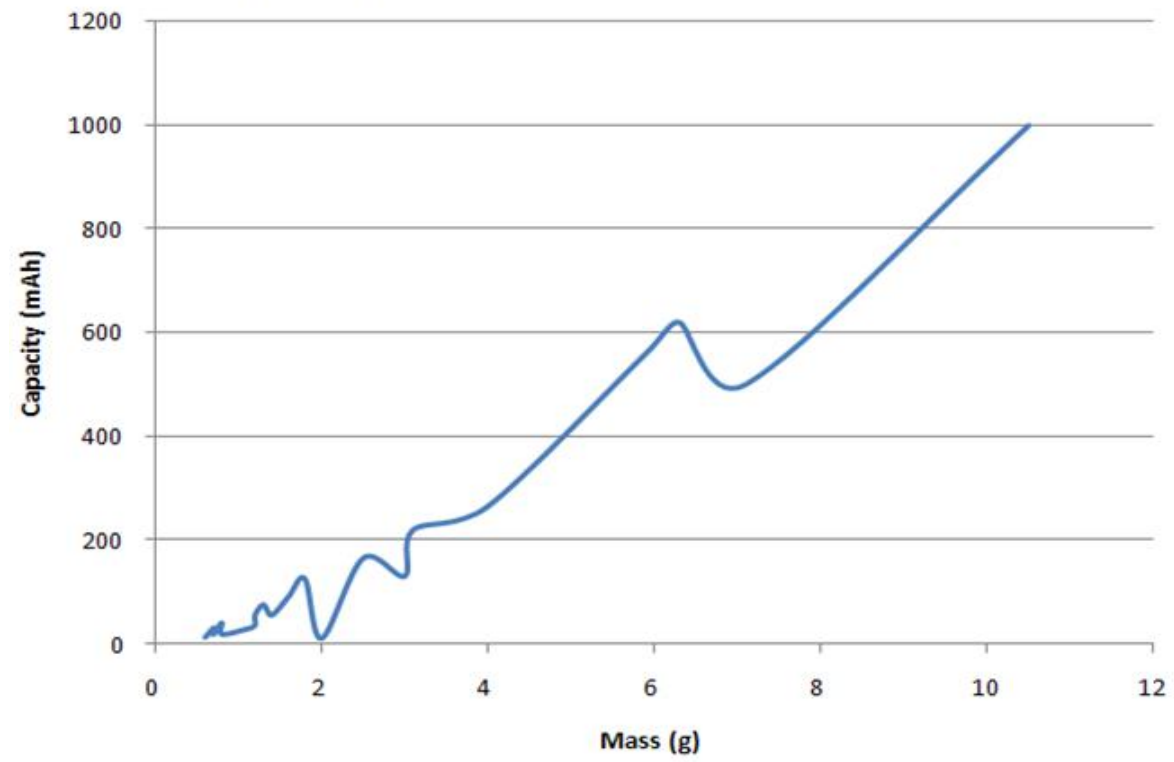

Fig. 6. Battery capacity plotted against mass for the panasonic CR lithium manganese dioxide cell range 


\section{SUMMARY}

It is clear from the review that distal limb recovery of rhythm information is possible. Lynn [12] and Yang [13] have successfully recovered QRST ventricular electrical activity from the upper arm, showing that the signal is present, in some form, along the limb. Electrical noise is the confounding factor of recovery from the wrist, though it has been shown that the wrist may not be the most suitable place for a monitor of this type. A more stable platform may be the upper arm. That being the case, the techniques for successful recovery of rhythm information are in existence today.

The importance of high quality signal recording has been made clear. Low noise, high impedence differential amplifiers are becoming common place in the electronics world. Their reliability is increasing and size and cost are decreasing, all driven by demand from other sectors, mainly mobile electronics. A reduction in physical size and over all power consumption, allows the construction of an extremely efficient, low noise device, which is ideal for use in a bodyworn monitor.

Low noise recovery techniques deal with environmental and general system noise. The recovered signal is still subject to additionally recovered biological signals such as EMG. These are also considered to be 'noise', as they are unwanted in this particular application. The removal of such is made complex by the fact that the frequency domains of the desired signal and that of the noise overlap, a factor which precludes bandwidth narrowing as a sole method of filtering and post processing. A review of nonsyntactic algorithms showed that empirical mode decomposition and partial reconstruction can be used to improve the denoising of ECG signals recovered from the left arm. It is also worth noting that the syntactic approach, although successful has excessive latency and is therefore not suitable for dysrhythmia or complex morphology analysis in real time.

As with all mobile technology the limitations are mainly from battery power sources. The possible implementation of an energy harvesting technique could increase the operation time and reduce the amount of patient interaction with the device, providing a more stable recording platform.

\section{CONCLUSION}

Cardiac disease is a prevalent killer in the modern world. More worryingly, the root cause of sudden cardiac death in the young often goes undiagnosed due to the inconvenience of the currently available long term monitoring solutions. The propositions mentioned herein describe the possibility of producing a noninvasive long term monitor. The challenges presented are in the areas of positioning for comfort, electrode design, signal processing and power supply technology. All of which are discussed.

It is evident from the literature the current technological capacity is such that developing a long term, non invasive continuous monitoring device is possible.

\section{CONSENT}

All authors declare that 'written informed consent was obtained from the patient (or other approved parties) for publication of this case report and accompanying images.

\section{ETHICAL APPROVAL}

All authors hereby declare that all experiments have been examined and approved by the appropriate ethics committee and have therefore been performed in accordance with the ethical standards laid down in the 1964 Declaration of Helsinki."

The clinical study conducted by Lynn D. et al. (2013), had ethical approval from the Office of Research...(ORECNI), reference number 10/NIR02/6, date of favourable ethical opinion: 20/05/2010, sponsored by Southern Health and Social Care Trust.

\section{COMPETING INTERESTS}

Authors have declared that no competing interests exist.

\section{REFERENCES}

1. Papadakis M, Sharma S, Cox S, Sheppard MN, Panoulas VF, Behr ER. "The magnitude of sudden cardiac death in the young: a death certificate-based review in England and Wales." Europace. 2009; 11(10):1353-1358. 
2. Sami Viskin, MD et al. "The Response of the QT Interval to the Brief Tachycardia Provoked by Standing - A Bedside Test for Diagnosing Long QT Syndrome". American College of Cardiology. 2010;55(18):195561.

3. Lewis DC. "Predicting the future of health care," The Brown University Digest. of Addiction Theory \& Application. 1999; 18(4):12-16.

4. Gemperle F, Kasabach C, Stivoric J, Bauer M, Martin R. Design for wearability. In The Second International Symposium on Wearable Computers, (ppll6- 122). Los Alamitos, CA: IEEE Computer Society; 1998.

5. MHRA. Drug Safety Update; 2012. Available:http://www.mhra.gov.uk/Safetyinf ormation/DrugSafetyUpdate/CON152725 Last accessed 31/07/14

6. NHS. Atrial Fibrillation; 2013. Available:http://www.nhs.uk/conditions/Atri al-fibrillation/Pages/Introduction.aspx Last accessed 30/07/14.

7. Omron. HCG-801 User Manual; 2011.

Available:http://www.omronhealthcare.com/data/catalog/3/655/1/IMHCG-801-E\%2005-11-2011\%20EN.pdf, Last accessed 31/7/14

8. Joshi AK, Kowey PR, Prystowsky EN, Benditt DG, Cannom DS, Pratt CM, McNamara A, Sangrigoli RM. First experience with a Mobile Cardiac Outpatient Telemetry (MCOT) system for the diagnosis and management of cardiac arrhythmia. Am J Cardiol. 2005;95:878881.

9. Zimetbaum P, Goldman A. Ambulatory Arrhythmia Monitoring: Choosing the Right Device. Circulation. 2010;122(1):16291636.

10. Crouch MA et al. Clinical Relevance and Management of Drug-Related QT Interval Prolongation; 2003.

Available:http://www.medscape.com/viewa rticle/458868 3 Last accessed 31/07/14

11. Shackel B, Chidsy KD, Shipley P. The assessment of chair comfort. Ergonomics. 1969;12(2):269-306.

12. Lynn WD, et al. "Arm and wrist surface potential mapping for wearable ECG rhythm recording devices: a pilot clinical study". Journal of Physics: Conference Series. 2013;450(1):1-8.

13. Yang $H$, et al. Study of Single-Arm Electrode for ECG Measurement Using Flexible Print Circuit; 2011.
Available:http://eshare.stust.edu.tw/Eshare File/2011 6/20116 7f351cdf.pdf, Last accessed 23/07/2014

14. Crone B. Common-Mode Rejection: How It relates to ECG subsystems and the techniques used to provide superior performance. Analogue Devices. MS-. 2011;2125 (1):1-4.

15. Horowitz $P$, Hill W. Precision circuits and low noise techniques. In: The Press Syndicate of the University of Cambridge The art of Electronics. 2nd ed. London: Cambridge University Press. 1989;286-315.

16. Escalona OJ. "Analog implementation of the single fiducial point alignment technique for real-time high resolution ECG analysis in the P-R interval." In Proceedings of Computers in Cardiology 1998, Cleveland, OH, USA. 1998;229-232.

17. William Craelius, et al. 'Criteria for optimal averaging of cardiac signals'. IEEE Transactions on Biomedical Engineering; 1986. BME No10.

18. Huang NE, et al. 'The empirical mode decomposition and Hilbert spectrum for non linear and non stationary time series analysis'. Proceeding of The Royal Society London. 1998;454.

19. Zhao Zhidong, et al. 'Adaptive noise removal of ECG signal based on ensemble empirical mode decomposition'. Hangzhou Dianzi University, China; 2011.

20. Lynn WD, et al. 'A Low Latency Electrocardiographic QRS Activity Recovery Technique for Use on the Upper Left Arm'. Electronics. 2014;3030409(3): 409-418.

21. Wu ZH, et al. 'Ensemble Empirical Mode Decomposition: A noise assisted data analysis method'. Advances in adaptive data analysis. 2009;1-40.

22. Mikhled Alfaouri, et al. 'ECG Signal denoising by wavelet transform thresholding'. Communication and electronics engineering Dept, Philadelphia University, Jordan. 2008;276-280.

23. Singh BN, et al. 'Optmal selection of wavelet basis functions applied to ECG signal denoising'. Digital Signal Processing. 2006;16:275-287.

24. Bahoura M, et al. 'DSP implementation of wavelet transform for realtime ECG waveforms detection and heart rate analysis', Computer Methods and Programs in Biomedicine. 1997;52:35-44. 
25. Pan J, Tompkins WJ. 'A realtime QRS detection algorithm'. IEEE Transcript Biomedical Engineering. 1985;32:230-236.

26. Righter, William $\mathrm{H}$, et al. US Patent 4938228, 'Wrist worn heart rate monitor'; 1990.

27. Escalona OJ, Mitchell $\mathrm{RH}$. 'Frequency bandwidth limitations in the signalaveraged ECG by the maximum coherence matching technique'. Automedica. 1991;13:177-186.

28. Nagel, JH. 'The Biomedical Engineering Handbook: Second Edition', CRC Press LLC, C70.1 - 70.2; 2000.

29. Webster JG. 'Medical Instrumentation, Application and Design, second edition', Houghton Mifflin Co, Boston, MA; 1992.

30. Wayne J. Smith, et al. 'Non-contact Biopotential Measurement from the Human Body Using a Low-Impedance Charge Amplifier'. Dept of Electrical and Computer Engineering, Biomedical Laboratory, Durham, NH; 2004.

31. Thomas J. Sullivan, et al. 'A Low Noise, Non-Contact EEG/ECG Sensor'. Division of Biological Sciences, University of California, San Diego, CA; 2007.

32. Plessey Semiconductors, Application note PS25201B 'EPIC Ultra High Impedance Electrophysiological Sensor', Swindon, UK; 2012.

Available:www.plesseysemiconductors.co m Accessed July 2014

33. Plessey Semiconductors, Press Release, 'Plessey uses its EPIC technology to create a heart monitor in a wrist watch', Swindon, UK; 2012.

Available:www.plesseysemiconductors.co $\mathrm{m}$, Accessed July 2014

34. Plessey Semiconductors, Application note 291465 'ECG using wrist mounted EPIC sensors', Swindon, UK; 2012.
Available:www.plesseysemiconductors.co m, Accessed July 2014

35. Lynn WD, et al. US Patent 0178902A1 'Belt Monitoring Systems and Methods', Schrader Electronics Ltd., UK; 2009.

36. Micro Power Battery Company. Panasonic Lithium Coin Batteries Tech Specs; 2014. Available:http://www.microbattery.com/spe cs-panasonic-lithium-coin Last accessed 31/07/14

37. Mallela VS, et al. 'Trends in Cardiac Pacemaker Batteries'. Indian Pacing and Electrophysiology Journal - Technical Series. 2004;4(4):201-212.

38. Lynn WD. US Patent 0316525A1 'Power transmission monitoring and maintenance systems and methods', Schrader Electronics Ltd., UK; 2011.

39. Maxwell Donelan, J, Naing, V, Li, Q. Biomechanical Energy Harvesting, Proceedings of Power MEMS'08, Sendai, Japan. 2008;39-44.

40. Feenstraa J, Granstroma J, Sodano H. Energy harvesting through a backpack employing a mechanically amplified piezoelectric stack. Mechanical Systems and Signal Processing. 2008;22(3):721734

41. Leonov V, Van Hoof C, Vullers RJM. Thermoelectric and hybrid generators in wearable devices and clothes. proceedings of body sensors networks. Berkeley, USA. 2009;195-200

42. Ozcanli OC. Turning body heat into electricity; 2010.

Available:http://www.forbes.com/2010/06/0 7/nanotech-body-heat-technologybreakthroughs-devices.html Last accessed $31 / 07 / 14$

(c) 2015 Lynn et al.; This is an Open Access article distributed under the terms of the Creative Commons Attribution License (http://creativecommons.org/licenses/by/4.0), which permits unrestricted use, distribution, and reproduction in any medium, provided the original work is properly cited.

Peer-review history:

The peer review history for this paper can be accessed here: http://www.sciencedomain.org/review-history.php?iid=842\&id=26\&aid=8835 